translational methods to solve population health problems in both health systems and in public health.

3073

Development and implementation of a faith-based community Health Advocate training programme for type-2 diabetes remission: A model for community-based non-communicable disease control

Kim Qumby ${ }^{1}$, Natasha Sobers, Natalie Greaves and Alafia Samuels ${ }^{1}$ The University of the West Indies

OBJECTIVES/SPECIFIC AIMS: The aim of this study is two-fold (1) to include a participatory action research design in the development of a community-based health advocate (HA) training programme which empowers community members to support the Barbados diabetes remission study 2 protocol - a low-calorie intervention for T2DM remission (2) to study the implementation of this programme with in select faith-based organisations (FBOs) which will act as community hubs. METHODS/STUDY POPULATION: Translation was informed using the RE-AIM framework. The target population were members of select FBOs. We assessed the readiness of FBOs to become community hubs in relation to human resources (the HA team must include someone with a clinical degree), infrastructure capacity (a private room for interviews) and the perspective (knowledge and attitude) of the FBO leadership to both the training and diabetes remission programmes. An open recruitment for HAs was made to the FBO membership and all who responded were accepted to the programme; which consisted of 8 weeks of face to face sessions inclusive of lectures and practical demonstrations and exercises specific to NCDs e.g. assessment of basic clinical parameters, ethics and nutrition. This was followed by a simulation exercise and a formal objective structured clinical examination (OSCE). HAs will participate in focus groups aimed at exploring the barriers and facilitators to the use of social media as a support system; this will be followed by participatory design workshops where the HAs will design support systems, inclusive of social media support, to assist participants in the diabetes remission intervention. RESULTS/ ANTICIPATED RESULTS: All three FBOs that were approached responded favourably and the programme was described as 'necessary' and 'timely' by the leadership. The FBOs were assessed and found to be ready. Thirty-eight persons signed up for the programme (more than the 21 we anticipated); 31 (82\%) attended at least 1 session and 29 completed the training; this indicates that implementation in the community is feasible. All who completed the programme attained an overall passing grade indicating the effectiveness of, and fidelity to the training. Initial feedback on the programme from HAs and volunteers indicates that it was acceptable. DISCUSSION/ SIGNIFICANCE OF IMPACT: This community-based training programme was successful in terms of reach, as both the FBO and the individual HA responded favourably; and effectiveness as measured by the expanded skill set of the HA. Initial feedback suggests that implementation of the programme is feasible in the community and acceptable to the HAs. Although this model focusses on diabetes remission utilising FBOs as hubs, it can be easily adapted to other NCDs e.g. hypertension and mental health; other disciplines e.g. surveillance; and other communities e.g. workplaces, homeless shelters.
Engagement in Out-Patient Services among Pregnant and Postpartum Women with Opioid Addiction: A Qualitative Study

Elizabeth Peacock-Chambers ${ }^{1}$, Mary T. Paterno, PhD CNM, Daniel Kiely, Tinamarie Fioroni and Peter D. Friedmann, MD, MPH ${ }^{1}$ Tufts University

OBJECTIVES/SPECIFIC AIMS: This study aims to understand the factors influencing engagement with out-patient services from pregnancy to 1 year postpartum among women in recovery from opioid use disorders (OUD). METHODS/STUDY POPULATION: We conducted semi-structured qualitative interviews and a brief survey with 20 mothers in OUD recovery recruited from health care and community organizations in Western MA. Transcripts were coded by two independent coders and analyzed using a qualitative descriptive approach. RESULTS/ANTICIPATED RESULTS: The average duration of any addiction treatment among the 20 participants was 5.6 years with $80 \%$ receiving medication-assisted treatment during a pregnancy. Approximately two-thirds experienced relapse during pregnancy or the first year postpartum. We identified 3 themes elucidating women's experiences around service engagement: "How I see myself" (personal development), "How services see me" (service delivery quality), and "Are you with me?" Personal development included response to past trauma, coping strategies and self-advocacy, and adjusting to parenthood. Service delivery quality was influenced by service design, efficacy of individual providers, and cultural norms (organizational and societal). In the final theme, intersection of individual and service-level factors influenced the degree and quality of the women's experience engaging with services. DISCUSSION/ SIGNIFICANCE OF IMPACT: Women describe successful engagement when they experience service providers as being emotionally supportive, delivering relevant services, and advocating on their behalf. To best support and engage families affected by OUD, relevant and timely services should be linked with compassionate delivery.

3421

\section{Evaluation of novel biomarkers of hepatocellular carcinoma development and recurrence in liver transplant patients \\ Jenna Mancinelli ${ }^{1}$, David Walls ${ }^{1}$, Baoli Chang ${ }^{1}$, Brendan Keating ${ }^{1}$, Maarouf Hoitet ${ }^{1}$ and Abraham Shaked ${ }^{1}$ \\ ${ }^{1}$ University of Pennsylvania School of Medicine}

OBJECTIVES/SPECIFIC AIMS: Given the poor prognosis of HCC and its increasing incidence worldwide, identifying biomarkers of $\mathrm{HCC}$ has been an active area of research. While biomarkers are being identified at a rapid pace, many are still in early phases of clinical study and very few have proven clinical utility. The objective of this study is to identify novel biomarkers of HCC and evaluate their clinical utility as predictors of disease development and prognosis with specific emphasis on disease recurrence after liver transplantation. Biomarkers will be identified through GWAS, as well as through analysis of qualitative and quantitative liver traits by magnetic resonance imaging (MRI). These novel biomarkers will then by implemented into risk prediction models aimed to assess an individual's risk for development of HCC and stratify their level of risk according 